\title{
SUBMILLIMETER OBSERVATIONS OF AN ASYMMETRIC DUST DISK AROUND FOMALHAUT
}

\author{
W. S. Holland, J. S. Greaves, W. R. F. Dent, and M. C. Wyatt \\ UK Astronomy Technology Centre, Royal Observatory, Blackford Hill, Edinburgh EH9 3HJ, UK \\ B. ZUCKERMAN \\ Department of Physics and Astronomy, University of California, Los Angeles, CA 90095 \\ R. A. WEBB \\ NASA-Ames Research Center, Moffet Field, CA 94035 \\ C. MCCARTHY \\ Department of Terrestrial Magnetism, Carnegie Institution of Washington, 5241 Broad Branch Road NW, Washington, DC 20015 \\ I. M. Coulson AND E. I. Robson \\ Joint Astronomy Centre, 660 North A‘ohoku Place, University Park, Hilo, HI 96720 \\ AND \\ W. K. GEAR \\ Department of Physics and Astronomy, University of Wales, Cardiff, 5 The Parade, Cardiff CF24 3YB, UK \\ Received 2002 April 17; accepted 2002 September 20
}

\begin{abstract}
New submillimeter images of the cold dust emission around the nearby main-sequence star Fomalhaut are presented. Observations at a wavelength of $450 \mu \mathrm{m}$, where the telescope beam size is equivalent to a resolution of $50 \mathrm{AU}$, reveal that Fomalhaut is encircled by a significantly nonaxisymmetric inclined ring. Smooth axisymmetric models of the ring images suggest the existence of a least one "clump" with an estimated flux of about $5 \%$ of the total from the disk, thus implying a clump mass of 0.075 lunar masses. At the resolution of the data, this clump could instead be a ring arc. The most plausible explanation is that this feature is produced by dust trapped in a resonance with a large planet. The observed structures around Fomalhaut and other Vega-excess stars qualitatively resemble features seen in numerical simulations with a gas giant perturber.
\end{abstract}

Subject headings: circumstellar matter — planetary systems — stars: individual (Fomalhaut)

\section{INTRODUCTION}

Imaging debris disks of cold dust around nearby stars can give vital clues to the planetary formation process (e.g., Zuckerman 2001). This dust is thought to arise from material left over from the formation of planets, being continually replenished by collisions of comets and asteroids. Not only do such images give an effective "time series" showing how our early planetary system evolved from a circumstellar disk, but perturbations, seen as clumps and cavities in the observed image, have the potential for actually pinpointing the locations of young planets (Ozernoy et al. 2000; Liou et al. 2000). If planets do exist, it has been proposed that interplanetary dust will interact with these larger bodies while spiraling in toward the star, resulting in central clearing and irregular variations in both the density and the corresponding brightness distribution. Hence, the study of debris disks is a potentially powerful way to reveal the presence of large, unseen bodies such as planets.

Although the edge-on disk around $\beta$ Pic had been studied extensively in the optical and near-infrared (e.g., Kalas \& Jewitt 1995), it was not until 1998 that significant new results emerged for several other stars from mid-infrared and submillimeter studies (Holland et al. 1998; Jayawardhana et al. 1998; Koerner et al. 1998; Greaves et al. 1998). By imaging the faint thermal emission from cold dust grains, submillimeter-wave techniques can circumvent the difficulties of the starlight that dominates at optical and near-infrared wavelengths. The much hotter star has a relatively small surface area compared to the grains and so has very little flux at these long wavelengths. The submillimeter is also sensitive to the cool dust spectrum, and, as the emission is invariably optically thin, direct mass estimates can be obtained.

The submillimeter images of Vega, Fomalhaut, and $\epsilon$ Eri, taken at a wavelength of $850 \mu \mathrm{m}$, showed dust disks similar in size to the Sun's Kuiper belt of comets (Holland et al. 1998; Greaves et al. 1998) and revealed direct evidence of central cavities in the emission around at least two of the stars - possibly cleared out by the formation of planets. The best-studied case is that of Fomalhaut, which has a central cavity cleared of $90 \%$ of its dust (Dent et al. 2000). The existence of such cavities was previously and subsequently inferred from IR-submillimeter spectral energy distributions (Backmann \& Paresce 1993; Walker \& Heinrichsen 2000; Dent et al. 2000). Bright peaks in the dust morphology at Vega and $\epsilon$ Eri could also be caused by planetary perturbations, and by tracking the orbital motion of dust features, one can potentially trace planets of relatively low mass at large distances from the star.

In this paper we present new data at short submillimeter wavelengths $(450 \mu \mathrm{m})$ of the dust emission around the nearby main-sequence star $\alpha$ Piscis Austrinus (Fomalhaut). These observations were motivated by the relative brightness of the Fomalhaut disk, among stars lying within $10 \mathrm{pc}$. The close proximity allows features similar in size to our solar system to be resolved. This star is of type A3 V, 7.7 pc from the Sun, and lies on the young main sequence with an age of approximately $200 \mathrm{Myr}$ (Barrado y Navascues et al. 1997). The improved spatial resolution obtainable at 450 
$\mu \mathrm{m}$ (equivalent to $50 \mathrm{AU}$ at the distance of Fomalhaut) allows a better investigation of small-scale structure, such as asymmetries and "warps" that are potential signatures of planetary formation.

\section{OBSERVATIONS AND RESULTS}

The SCUBA bolometer camera (Holland et al. 1999) was used on the James Clerk Maxwell Telescope (JCMT) to obtain deep simultaneous images of Fomalhaut at wavelengths of 450 and $850 \mu \mathrm{m}$. SCUBA has two arrays of detectors containing 91 pixels at $450 \mu \mathrm{m}$ and 37 pixels at $850 \mu \mathrm{m}$, with a total field of view of approximately 2.4 in diameter at each wavelength. Each pixel has diffraction-limited resolution on the sky with beam sizes of 7".5 and 14" (FWHM) at 450 and $850 \mu \mathrm{m}$, respectively. During late 1999 SCUBA was upgraded with new (wideband) filters that improved the sensitivity by $50 \%$ and $15 \%$ pixel $^{-1}$ at 450 and $850 \mu \mathrm{m}$ through a combination of wider filter bandwidths and improved instrument transmission. In addition, the past year has seen considerable improvements in the calibration accuracy achievable at short submillimeter wavelengths (Archibald et al. 2002). The resulting data benefited from some of the highest quality submillimeter weather on Mauna Kea (mean zenith opacities of 1 at $450 \mu \mathrm{m}$ ) and a system substantially improved in both sensitivity and calibration accuracy.

The observing mode used was "jiggle-mapping," in which the secondary mirror of the telescope compensates for the instantaneous undersampling by offsetting the array position to produce a Nyquist-sampled image (Holland et al. 1999). This results in 364 and 148 independent samples on the sky per map at 450 and $850 \mu \mathrm{m}$. The final images result from a co-add of eight individual maps with a total integration time of $5.7 \mathrm{hr}$. Accurate telescope pointing was crucial to these observations and was checked before and after each map using the nearby blazar 2255-282 with rms error of less than $2^{\prime \prime}$. The data were reduced using the SURF software package (Jenness \& Lightfoot 1998). Flux calibration was obtained using beam maps of Uranus with calibration accuracies estimated to be about $10 \%$ and $5 \%$ at 450 and $850 \mu \mathrm{m}$, respectively. The general limit on characterizing the disk is the spatial resolution of the data. Very little information can be deduced about sizes or positions differing by $\approx 2^{\prime \prime}$, which at the distance of Fomalhaut corresponds to $15 \mathrm{AU}$, or $5^{\circ}$ of angle within the disk.

The 450 and $850 \mu \mathrm{m}$ images of Fomalhaut are shown in Figures $1 a$ and $1 b$ with flux estimates detailed in Table 1 . The $850 \mu \mathrm{m}$ image is consistent with the previously pub-
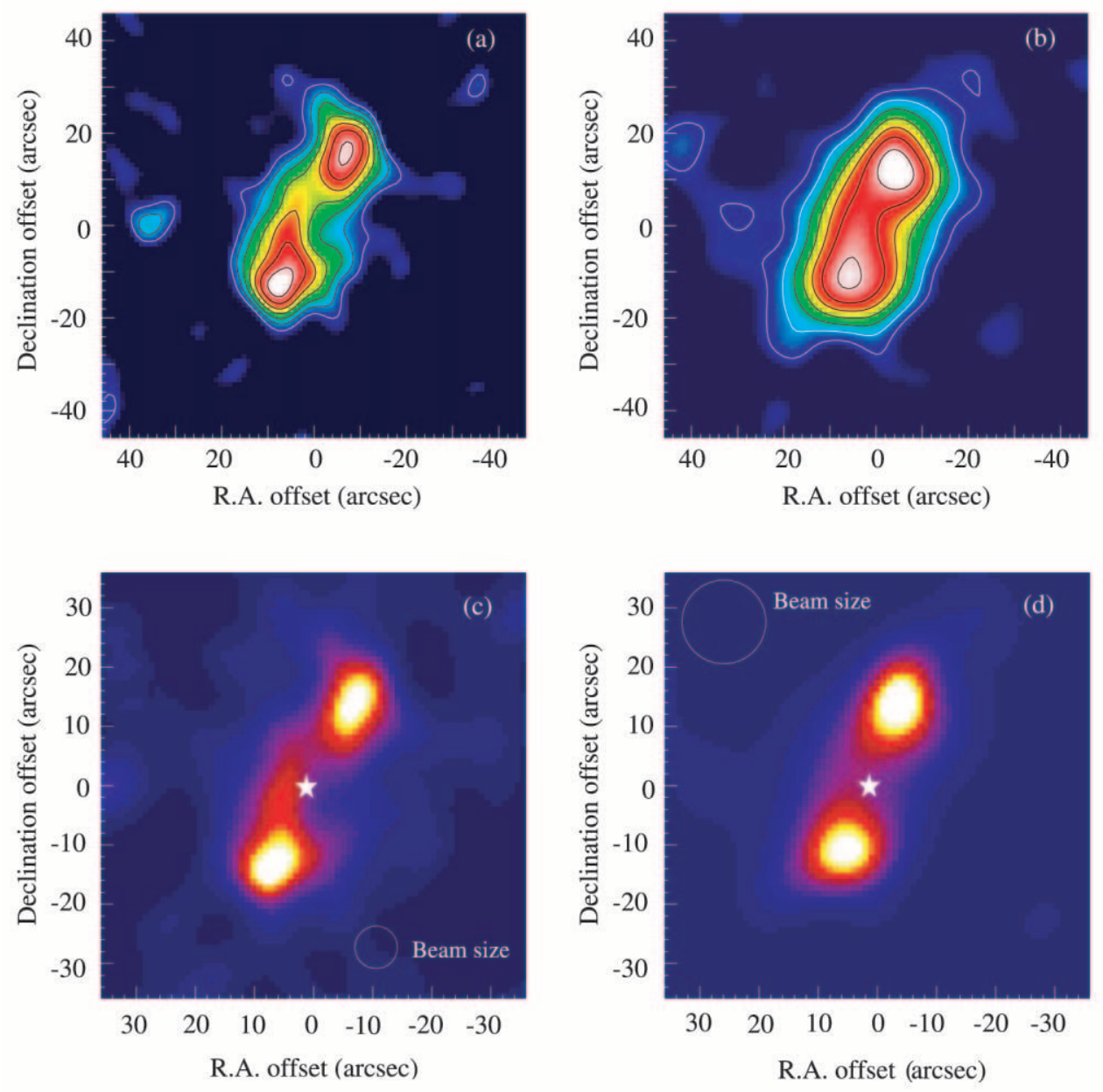

FIG. 1. - 450/850 $\mu \mathrm{m}$ images of the dust emission around Fomalhaut. North is up and east is to the left. The R.A. and declination offsets are relative to the J2000 position of Fomalhaut (R.A. $22^{\mathrm{h}} 57^{\mathrm{m}} 39^{\mathrm{s}} 0$, decl. $-29^{\circ} 37^{\prime} 20^{\prime \prime}$; ; proper motion $329 /-164$ mas $\mathrm{yr}^{-1}$ ). $(a, b)$ " Raw " images smoothed with a $7^{\prime \prime}$ Gaussian to improve the signal-to-noise ratio. The contours start at $3 \sigma$ and increase in $1 \sigma$ steps for the $450 \mu \mathrm{m}$ and $2 \sigma$ steps for the $850 \mu \mathrm{m}$ image. After smoothing, the individual map $1 \sigma$ contour levels are 13 and $2.0 \mathrm{mJy}$ beam $^{-1}$ at 450 and $850 \mu \mathrm{m}$, respectively. $(c)$ and $(d)$ have been deconvolved with the measured telescope point-spread function using the Richardson-Lucy deconvolution technique (see Smith et al. 2000 for details of this technique applied to SCUBA data). The stellar position is marked by the star symbol. The resolution of the beam (FWHM of 7..5 at 450 and $14^{\prime \prime}$ at $850 \mu \mathrm{m}$ ) is also shown for scaling purposes. 
TABLE 1

OBSERVATION SUMMARY

\begin{tabular}{|c|c|c|c|c|c|}
\hline $\begin{array}{l}\text { Wavelength } \\
\qquad(\mu \mathrm{m})\end{array}$ & 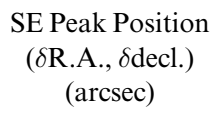 & $\begin{array}{c}\text { Flux }^{\mathrm{a}} \\
\left(\mathrm{mJy} \mathrm{beam}^{-1}\right)\end{array}$ & $\begin{array}{l}\text { NW Peak Position } \\
\text { ( } \delta \text { R.A., } \delta \text { decl.) } \\
(\operatorname{arcsec})\end{array}$ & $\begin{array}{c}\text { Flux }^{\mathrm{a}} \\
\left(\mathrm{mJy} \mathrm{beam}^{-1}\right)\end{array}$ & $\begin{array}{l}\text { Integrated Flux } \\
(\mathrm{mJy})\end{array}$ \\
\hline .............. & $7.1,-12.3$ & $119 \pm 20$ & $7.4,-16.2$ & $124 \pm 20$ & $595 \pm 35$ \\
\hline $850 \ldots \ldots \ldots \ldots \ldots$ & $5.9,-10.9$ & $27.0 \pm 3.0$ & $-4.3,12.1$ & $29.4 \pm 3.0$ & $97 \pm 5$ \\
\hline
\end{tabular}

a The fluxes are measured from the raw (nonsmoothed) maps.

b The integrated fluxes are estimated within an aperture of radius $30^{\prime \prime}$ centered on the star and have been corrected for the telescope error beam according to Sandell 1997.

lished data (Holland et al. 1998), showing an elongated structure at a position angle of $160^{\circ}$-interpreted as a roughly edge-on torus. Although the north peak appears slightly brighter than the southern counterpart, contrary to the image of Holland et al. (1998), the difference is not significant $(<1 \sigma)$. Likewise, the lobe shapes being slightly elliptical in different directions is not significant within the limits of the spatial resolution.

The central dip in the emission results from the geometric effect of a lower column density of grains seen toward the star. However, the $450 \mu \mathrm{m}$ image, benefiting from 7."5 resolution (equivalent to $50 \mathrm{AU}$ ), reveals a distinct bend in the connecting emission between the two offset peaks. That is, after the lobes the next brightest emission is not located symmetrically between the peaks but to one side (east). The effect is just barely visible in the lower resolution $850 \mu \mathrm{m}$ image, where there is a "notch" at the stellar position and more emission to the east side. This lends support to the reality of the nonaxisymmetric dust distribution. In addition, to verify that the observed structure is not associated with any artifacts of the telescope beam pattern, which contains a significant sidelobe power contribution at $450 \mu \mathrm{m}$, the data were deconvolved with the measured telescope point-spread function. The resulting images in Figures $1 c$ and $1 d$ clearly show that the bend in emission toward the east side of the disk is real.

\section{ANALYSIS}

\subsection{Nonuniformity of the Ring}

The images confirm that we are looking at an almost edge-on dust disk or ring, with a cavity extending out to approximately $100 \mathrm{AU}$ radius from the star. The inclination of the disk plane to the plane of the sky, derived from the axial ratio of the full emission extent (to the $3 \sigma$ level) in the deconvolved image in Figure $1 c$ is $70^{\circ} \pm 4^{\circ}$, consistent with previous estimates at $100 \mu \mathrm{m}$ (Lester et al. 1989) and at 850 $\mu \mathrm{m}$ (Holland et al. 1998). The apparent width across the center of the ring is $\approx 30^{\prime \prime}$ (at the $3 \sigma$ contour), whereas the outer radius and inclination predict a thin-disk width of up to $20^{\prime \prime}$. This difference $\left(10^{\prime \prime}\right)$ implies that the vertical thickness of the ring is $\approx 80 \mathrm{AU}$, slightly less than the $120 \mathrm{AU}$ estimated by Dent et al. (2000) from the previous lower resolution $850 \mu \mathrm{m}$ data.

The two bright peaks result from looking tangential to the ring where the effective column density is largest. However, the emission that connects the lobes is not smoothly distributed around an ellipse centered on the star. In Figure 2 the flux profile is plotted around an ellipse connecting the two bright lobes with a fitted axis that matches the observed structure as closely as possible. This clearly shows the main bright region of the ring to the east of the star and a large much fainter region to the west. The three simplest interpretations are that (1) there is a section of enhanced brightness to the east in an otherwise faint ring or (2) there is a section of weaker emission in the west side of an otherwise bright ring or (3) the ring is seen very close to edge-on and there is a separate source associated with the disk but above the midplane. Of these hypotheses, the first is the most likely; it is difficult dynamically to create a single gap in an otherwise uniform ring (option 2), and a separate clump above the ring plane (option 3 ) would presumably not have a stable orbit.

\subsection{Characterization of Structure in the Ring}

In an attempt to classify the structure further, the observed $450 \mu \mathrm{m}$ disk has been fitted with a smooth ring model. This model is described by the distribution of the orbital elements of the material in the ring, resulting in a uniform torus centered on the star (see Wyatt et al. 1999 for more details). The opening angle of this torus is defined by the mean inclination of the orbits, $I$, and the smoothly

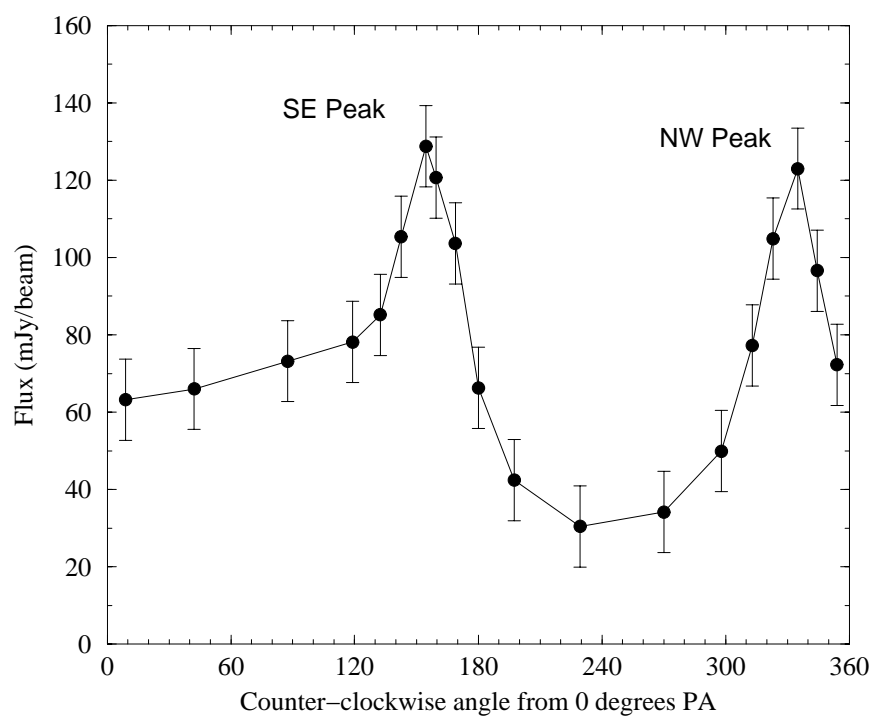

FIG. 2.-Flux profile connecting the two main lobes in the $450 \mu \mathrm{m}$ smoothed observational image (Fig. $1 a$ ) around an ellipse at $16^{\prime \prime}$ radius. The angular offset is measured as a position angle from the star position in the conventional sense (i.e., counterclockwise from north), and the signal is measured in beam-sized circles at half-beam increments. The increments are equal angles as if the disk were observed face-on. The error bars represent the $3 \sigma$ uncertainty in each measurement based on the rms noise in the image. 

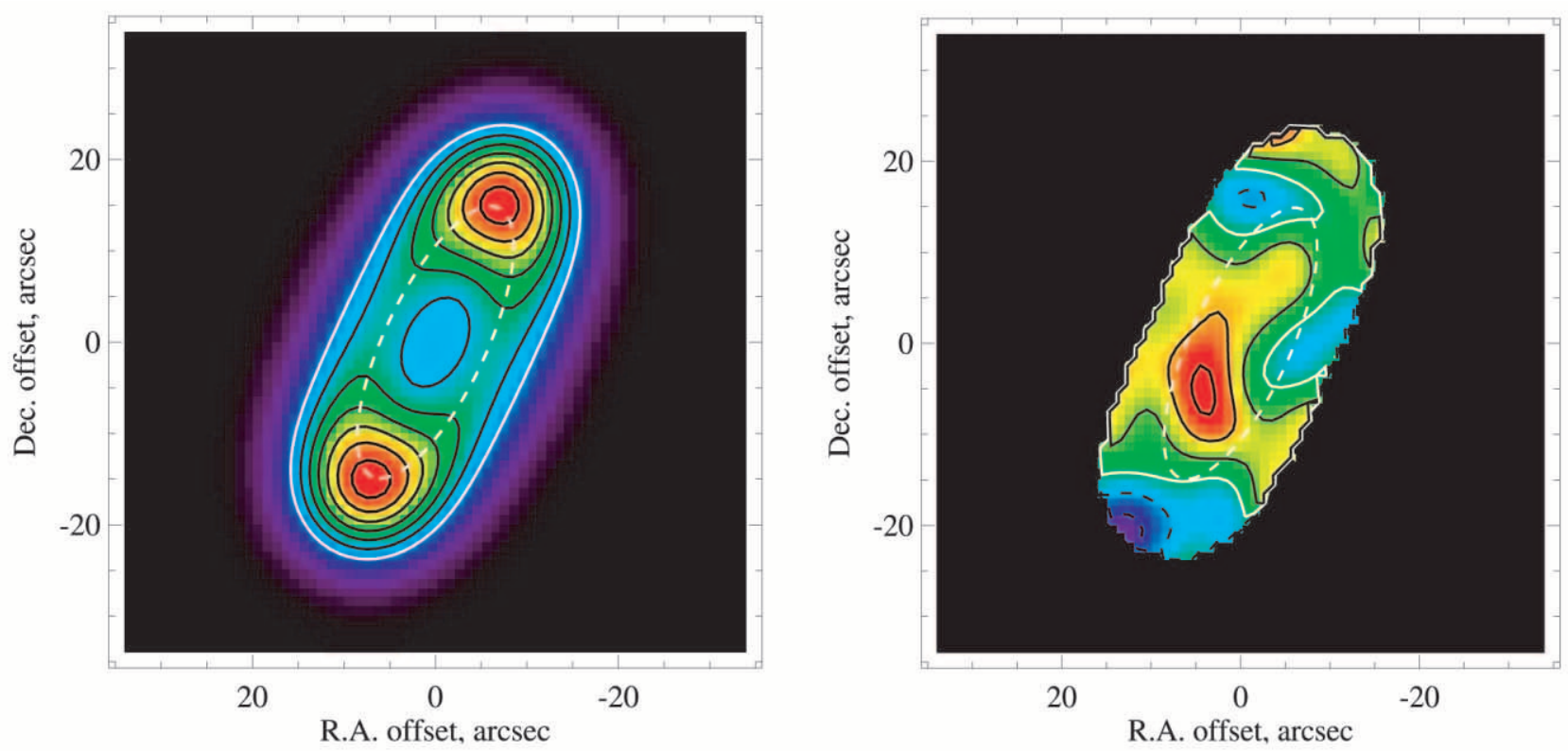

FIG. 3.-Left: Axisymmetric smooth disk model based on the $450 \mu \mathrm{m}$ image. Right: Residual image (observed - smooth model), which shows that the asymmetry seen in the observed data could be explained by a clump embedded in an otherwise smooth disk. All contours are spaced at $1 \sigma=13 \mathrm{mJy}$ beam ${ }^{-1}$. For the left panel, the lowest (white) contour is set at $3 \sigma$, while for the right panel, the (black) dashed line indicates the zero level and the solid lines are spaced by $1 \sigma$. The dashed white line on the images shows the approximate inner edge of the midplane of the disk, a $120 \mathrm{AU}$ radius ring inclined at $70^{\circ}$ to the plane of the sky.

varying radial distribution is defined by the orbits' mean eccentricities, $e$, their inner and outer cutoffs in semimajor axis, $a_{\min }$ and $a_{\max }$, and $\gamma$, the exponent in a power-law distribution of the semimajor axis, where $n(a) \propto a^{\gamma}$. The model fits a smooth ring to the faint west side emission as well as the main peaks and cavity. The fit quality was assessed by comparing the model results to three crosscuts through the image: along a line connecting the lobes and two perpendicular lines, one through each lobe.

Figure 3 (left) shows the axisymmetric smooth ring model for comparison with the Fomalhaut image (Fig. 1a). The model has $a_{\min }=125 \mathrm{AU}, a_{\max }=185 \mathrm{AU}, \gamma=-1, I=5^{\circ} .1$, $e=0.065$, and the ring plane inclined $70^{\circ}$ to the plane of the sky. While the cutoffs in semimajor axis are sharp, the eccentric dust orbits mean that the inner and outer edges of the model ring are smooth. However, most material in the model is confined between 125 and 185 AU with a peak density at about $135 \mathrm{AU}$ radius. This corresponds to a $35^{\prime \prime}$ diameter, slightly farther apart than the $32^{\prime \prime}$ separation of the peaks at $450 \mu \mathrm{m}$ (Table 1); as noted by Dent et al. (2000) the limited resolution of the data tends to reduce the depth of the cavity and hence "draw in " the peaks.

The residual image (observed - model) shown in Figure 3 (right) suggests that the asymmetry could be explained by a bright clump or arc associated with the smooth disk. The clump is detected at the $3 \sigma$ level but may be more significant since it appears to extend over more than one beamwidth and is also seen in the $850 \mu \mathrm{m}$ image. The clump also appears to be inside the minimum model radius of $125 \mathrm{AU}$. Assuming that it lies in the midplane of the ring, this suggests that the clump is $\approx 100$ AU from the star. It is unresolved in the narrowest dimension plane but appears to be extended along the disk axis, with a deconvolved size of $\approx 8^{\prime \prime}$, or $60^{\circ}(\approx 50 \mathrm{AU})$ around the ring (see also Fig. 2). Thus, it may also form a partial arc of dust.

\subsection{Dust Properties}

Dust emission properties were modeled using Mie theory and a composition and size distribution for the dust grains optimized to best fit the spectral energy distribution of the disk emission (Wyatt \& Dent 2002). The best fit was found for nonporous grains composed of $\frac{1}{3}$ (by volume) amorphous silicate and $\frac{2}{3}$ refractory organics (Li \& Greenberg 1997). The dust distribution was fitted by $n(m) \propto m^{-1.84}$ (number per unit mass) down to $7 \mu \mathrm{m}$ diameter grains. Below this size, grains are expected to be blown out of the system by radiation pressure. This distribution might be produced in a collisional cascade (Wyatt \& Dent 2002). Grain temperature depends on size and location; at 125-175 AU large $(>1 \mathrm{~mm})$ grains have temperatures between 40 and $50 \mathrm{~K}$, while small $(7 \mu \mathrm{m})$ grains have temperatures between 65 and $75 \mathrm{~K}$.

The dust disk properties are similar to previous estimates, based mainly on $850 \mu \mathrm{m}$ data (Holland et al. 1998; Dent et al. 2000). The previous temperature estimate was slightly lower at $40 \mathrm{~K}$ (for an effective single size of grain), and the disk size was slightly smaller (inner and outer radii of 100 and $140 \mathrm{AU}$; Dent et al. 2000). Thus, the new and better constrained $450 \mu \mathrm{m}$ total flux has not significantly altered estimates of the disk properties, although the submillimeter opacity index, $\beta$, is revised slightly downward, from 1.1 to about 0.8 . This strengthens the evidence for a population of large grains approaching blackbody properties $(\beta=0)$. There are no significant variations of $\beta$ across the disk, within the errors. Following the method described in Holland et al. (1998), we estimate the clump mass to be 0.075 lunar masses (or approximately $5 \%$ of the total disk mass, which remains unchanged at 1.5 lunar masses). However, this value is highly dependent on the populations of larger dust grains (Wyatt \& Dent 2002). The possible range in 
emissivity values of a factor of 4 is discussed by Greaves at al. (1998).

\section{DISCUSSION}

The dust around Fomalhaut is evidently nonaxisymmetric in the region between the end lobes of the torus. A nonunique smooth ring model suggests the existence of a clump or an arc in the underlying emission near the star. We have considered various explanations for the brightness asymmetry seen in Figure 1, concluding that it originates in a clump associated with, or just inside, the main disk.

It was shown by Wyatt et al. (1999) that the gravitational perturbations of an external stellar companion, or an internal planetary system, could cause the dust disk to have an asymmetric brightness distribution. Their pericenter glow model showed how an eccentric perturber imposes a forced eccentricity on the orbit of the disk particles, causing the disk to have a center of symmetry offset from the star and resulting in the closer side (i.e., the forced pericenter side) being hotter and therefore brighter than the opposite side. This model was used to explain the brightness asymmetry observed in the HR 4796A disk (Telesco et al. 2000). The same modeling technique was applied to Fomalhaut in an attempt to explain the structure in the submillimeter images. However, to reproduce the glow in the bright east section of the ring, the forced asymmetry becomes too large $(e>0.6)$. This would result in an increase in brightness of the southern lobe (nearest to the clump) by a factor of 2 greater than the northern end, whereas they are observed to be equal in brightness to better than $10 \%$ (as shown in Table 1).

An alternative hypothesis is that the ring has genuine nonazimuthal structure due to dust trapped in mean motion resonances with a planet. Recent work suggests that a planet, via resonances and gravitational scattering, produces an asymmetric dust belt with one or more unevenly spaced clumps, as well as a central cavity void of dust emission (Liou \& Zook 1999; Ozernoy et al. 2000; Liou et al. 2000). The resonant effect has in fact been seen in the solar system, in the dust ring lying along the Earth's orbit (Dermott et al. 1994). Such techniques are potentially very powerful in identifying signatures of exosolar planets embedded in debris dust disks. The initial numerical simulations, performed by Ozernoy et al. (2000), showed a remarkable similarity to the observed structures surrounding Vega and $\epsilon$ Eri.

Such detailed simulations have not yet been performed on the Fomalhaut disk. However, it has been shown that dust trapped in mean motion resonance $1: 1$ with a $0.3 M_{\mathrm{J}}$ planet in a circular orbit can produce horseshoe or arclike structures of the type observed around Fomalhaut (Ozernoy et al. 2000). Changing the planet mass significantly affects the structure; for example, morphologically different structures are produced by Earth- and Saturn-like planets (although the exact values will be slightly modified for different stellar masses). The stellar mass is most significant for affecting orbital timescales. The fraction of perturbed dust surrounding Fomalhaut is similar to that seen around Vega and $\epsilon$ Eri (Holland et al. 1998; Greaves et al. 1998). As in the case of $\epsilon$ Eri, if this is indeed evidence for planetary companions, then they would be at significantly larger radii from the star $(\approx 50-100 \mathrm{AU})$ than observed in our own solar system. It remains unclear whether massive planets can form at such large distances from stars.

Other models for producing asymmetric clumps are very implausible - generating local dust in a collision would require destroying bodies with a combined mass of half that of Pluto (0.075 lunar masses; Wyatt \& Dent 2002), while a perturbing flyby by another star would have to have occurred within the very recent past (of order $50,000 \mathrm{yr}$; Larwood \& Kalas 2001), and no such star has been identified (Deltorn \& Kalas 2001). One final possibility is that the clump is not associated with the disk at all and is in fact a background object such as a high- $z$ galaxy, in chance alignment with the disk. The clump has an estimated flux of 30 and $5 \mathrm{mJy}$ at 450 and $850 \mu \mathrm{m}$, respectively, and our observations of debris disks typically identify one source per field at this flux level. This translates to a source count of about $0.25 \mathrm{arcmin}^{-2}$ - consistent with the deep SCUBA extragalactic surveys (see, e.g., Blain et al. 1999). However, the statistical probability of finding such a galaxy offset by up to $10^{\prime \prime}$ from the star is only $2.5 \%$, so it is unlikely that the clump is a background object.

\section{CONCLUSIONS}

We have presented new, high signal-to-noise ratio submillimeter images of a dusty disk surrounding the young main-sequence star Fomalhaut. The disk is close to edge-on to the line of sight, and our $450 \mu \mathrm{m}$ image, benefiting from $50 \mathrm{AU}$ resolution at the distance of the star, reveals the dust emission to be significantly asymmetric.

Interpretation of the observed structure remains elusive. Perhaps the most plausible explanation is that a clump or arc is produced by dust trapped in a resonance with a large planet. Numerical simulations of planets in the Vega and $\epsilon$ Eri systems show similarity to the observed disk structure. In addition, high-resolution millimeter-wavelength interferometry of Vega (Koerner, Sargent, \& Ostroff 2001; Wilner et al. 2002) has confirmed the structure originally reported by Holland et al. (1998). The spatial resolution achievable with interferometers (and particularly the next-generation facilities such as the Atacama Large Millimeter Array) is such that orbital motions may be measurable in the coming decade. It is clear that the clump in the Fomalhaut disk is not unique; almost all debris disks show clumps or arcs at a few percent level. It is becoming increasingly apparent that these structures are as common as the central cavities seen in the dust emission around nearby stars. Both phenomena are plausibly created by planetary systems.

The JCMT is operated by the Joint Astronomy Centre, on behalf of the UK Particle Physics and Astronomy Research Council, the Netherlands Organisation for Pure Research, and the National Research Council of Canada. This research was supported in part by PPARC funding, the UCLA Astrobiology Institute, and by a NASA grant to UCLA. 


\section{REFERENCES}

Archibald, E. N., et al. 2002, MNRAS, 336, 1

Backmann, D. E., \& Paresce, F. 1993, in Protostars and Planets III, ed. E. H. Levy \& J. I. Lunine (Tucson: Univ. Arizona Press), 1253

Barrado y Navascues, D., Stauffer, J. R., Hartmann, L., \& Balachandran, S. C. 1997, ApJ, 475, 313

Blain, A. W., Kneib, J.-P., Ivison, R. J., \& Smail, I. 1999, ApJ, 512, L87

Deltorn, J.-M., \& Kalas, P. 2001, in ASP Conf. Ser. 244, Young Stars near Earth: Progress and Prospects, ed. R. Jayawardhana \& T. Greene (San Francisco: ASP), 227

Dent, W. R. F., Walker, H. J., Holland, W. S., \& Greaves, J. S. 2000 , MNRAS, 314, 702

Dermott, S. F., Jayaraman, S., Xu, Y. L., Gustafson, B. A. S., \& Liou, J.-C. 1994, Nature, 369, 719

Greaves, J. S., et al. 1998, ApJ, 506, L133

Holland, W. S., et al. 1998, Nature, 392, 788 1999, MNRAS, 303, 659

Jayawardhana, R., Fisher, S., Hartmann, L., Telesco, C., Pina, R., \& Fazio, G. 1998, ÄpJ, 503, L79

Jenness, T., \& Lightfoot, J. F. 1998, in ASP Conf. Ser. 145, Astronomical Data Analysis Software and Systems VII, ed. R. Albrecht, R. N. Hook, \& H. A. Bushouse (San Francisco: ASP), 216

Kalas, P. \& Jewitt, D. 1995, AJ, 110, 794

Koerner, D. W., Ressler, M. E., Werner, M. W., \& Backmann, D. E. 1998, ApJ, 503, L83
Koerner, D. W., Sargent, A. I., \& Ostroff, N. A. 2001, ApJ, 560, L181

Larwood, J., \& Kalas, P. 2001, MNRAS, 323, 402

Lester, D., Harvey, P., Smith, B., Colomé, C., \& Low, F. L. 1989, BAAS, 21,1085

Li, A., \& Greenberg, J. M. 1997, A\&A, 323, 566

Liou, J.-C., \& Zook, H. A. 1999, AJ, 118, 580

Liou, J.-C., Zook, H. A., Greaves, J. S., \& Holland, W. S. 2000, in Lunar and Planetary Science XXXI, ed. C. Agee \& D. Black (Houston: LPI), abstract 1416

Ozernoy, L. M., Gorkavyi, N. N., Mather, J. C., \& Taidakova, T. A. 2000, ApJ, 537, L147

Sandell, G. H. L. 1997, Starlink Cookbook 11 (Hilo: Joint Astronomy Centre)

Smith, K. W., Bonnell, I. A., Emerson, J. P., \& Jenness, T. 2000, MNRAS, 319, 991

Telesco, C. M., et al. 2000, ApJ, 530, 329

Walker, H. J., \& Heinrichsen, I. 2000, Icarus, 143, 147

Wilner, D. J., Holman, M. J., Kuchner, M. J., \& Ho, P. T. P. 2002, ApJ, $569, \mathrm{~L} 115$

Wyatt, M. C., \& Dent, W. R. F. 2002, MNRAS, 334, 589

Wyatt, M. C., Dermott, S. F., Telesco, C. M., Fisher, R. S., Grogan, K., Holmes, E. K., \& Pina, R. K. 1999, ApJ, 527, 918

Zuckerman, B. 2001, ARA\&A, 39, 549 